Audiology Neurotology
Audiol Neurotol 2016;21:391-398

DOI: $10.1159 / 000455058$
Received: February 19, 2016

Accepted after revision: December 12, 2016

Published online: March 21, 2017

\title{
Towards a Unified Testing Framework for Single-Sided Deafness Studies: A Consensus Paper
}

\author{
Paul Van de Heyning ${ }^{a, b}$ Dayse Távora-Vieira ${ }^{c, d}$ Griet Mertens ${ }^{a, b}$ Vincent Van Rompaey ${ }^{a, b}$ \\ Gunesh P. Rajan $^{c, d}$ Joachim Müller ${ }^{\mathrm{e}}$ John Martin Hempel ${ }^{\mathrm{e}}$ Daniel Leander ${ }^{\mathrm{e}}$ Daniel Polterauer ${ }^{\mathrm{e}}$ \\ Mathieu Marx ${ }^{f}$ Shin-ichi Usami $^{g}$ Ryosuke Kitoh ${ }^{g}$ Maiko Miyagawa ${ }^{\text {h }}$ Hideaki Moteki $^{9}$ \\ Kari Smilskyi Wolf-Dieter Baumgartner' Thomas Georg Keintzelk Georg Mathias Sprinzl' \\ Astrid Wolf-Magele ${ }^{l}$ Susan Arndt ${ }^{m}$ Thomas Wesarg ${ }^{m}$ Stefan Zirn ${ }^{m, n}$ Uwe Baumann ${ }^{\circ}$ \\ Tobias Weissgerber $^{\circ}$ Tobias Rader $^{p}$ Rudolf Hagen ${ }^{q}$ Anja Kurz ${ }^{q}$ Kristen Rak $^{q}$ Robert Stokroos ${ }^{r}$ \\ Erwin George $^{r}$ Ruben Polo ${ }^{s}$ María del Mar Medina ${ }^{s}$ Yael Henkin ${ }^{t}$ Ohad Hilly ${ }^{u}$ David Ulanovski ${ }^{u}$ \\ Ranjith Rajeswaran ${ }^{v}$ Mohan Kameswaran ${ }^{\vee}$ Maria Fernanda Di Gregoriow ${ }^{\text {w }}$ Mario E. Zernotti ${ }^{x}$
}

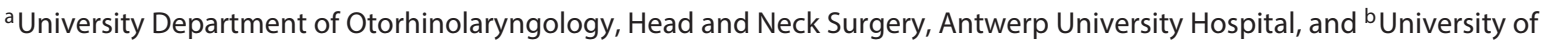
Antwerp, Antwerp, Belgium; ' Otolaryngology, Head and Neck Surgery, School of Surgery, University of Western Australia, and ${ }^{d}$ Fiona Stanley Hospital, Perth, WA, Australia; ${ }^{e}$ Ear, Nose and Throat Clinic and Policlinic, Ludwig Maximilian University, Munich, Germany; ${ }^{\mathrm{f} D e p a r t m e n t}$ of Otology-Neurotology and Skull Base Surgery, Hôpital Purpan, Toulouse, France; ${ }^{g}$ Otorhinolaryngology and h Hearing Implant Sciences, Shinshu University School of Medicine, Matsumoto, Japan; 'Department of Otolaryngology, Head and Neck Surgery, Sunnybrook Health Sciences Centre, University of Toronto, Toronto, ON, Canada; 'Ear, Nose and Throat Department, University Clinic Vienna, Vienna, k Department of Otorhinolaryngology, Klinikum Wels-Grieskirchen GmbH, Wels, and 'Department of Otorhinolaryngology, University Clinic St. Pölten, St. Pölten, Austria; ${ }^{m}$ Department of Otorhinolaryngology, Head and Neck Surgery, University Medical Center Freiburg, Freiburg, nUniversity of Applied Sciences Offenburg, Faculty of Electrical Engineering and Information Engineering, Offenburg,

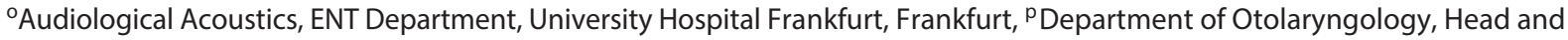
Neck Surgery, University of Mainz, Mainz, and 'Department of Otolaryngology, University Hospital Würzburg, Würzburg, Germany; ' Department of Otorhinolaryngology, Head and Neck Surgery, Maastricht University Medical Center, Maastricht, The Netherlands; 'Stolaryngology and Head and Neck Surgery Department, Hospital Universitario Ramón y Cajal, Madrid, Spain; ${ }^{\mathrm{D}}$ Department of Communication Disorders, Sackler Faculty of Medicine, Tel Aviv University, Tel Aviv, and The Hearing, Speech \& Language Center, Sheba Medical Center, Tel Hashomer, and uPediatric ENT Unit, Schneider Children's Hospital, Petach Tikva and Tel Aviv University, Tel Aviv, Israel; " ${ }^{2}$ Madras ENT Research Foundation, Chennai, India; ${ }^{\text {w} D e p a r t m e n t ~ o f ~}$ Otoneurology, Sanatorio Allende, and ${ }^{x}$ Department of Otolaryngology, Catholic University of Córdoba, Córdoba, Argentina
\end{abstract}

\section{Keywords}

Single-sided deafness · Unilateral hearing loss · Asymmetric hearing loss · Bimodal stimulation · Cochlear implant . Contralateral routing of signals hearing aid . Bone-anchored hearing aid · Bone conduction device . Testing method consensus

\begin{abstract}
Background: While hearing aids for a contralateral routing of signals (CROS-HA) and bone conduction devices have been the traditional treatment for single-sided deafness (SSD) and asymmetric hearing loss (AHL), in recent years, cochlear implants ( $\mathrm{Cls}$ ) have increasingly become a viable treatment choice, particularly in countries where regulatory approval and reimbursement schemes are in place. Part of
\end{abstract}

\section{KARGER \\ E-Mail karger@karger.com} www.karger.com/aud

\section{(9) 2017 The Author(s) \\ Published by S. Karger AG, Basel 1420-3030/17/0216-0391\$39.50/0} This article is licensed under the Creative Commons Attribution-
NonCommercial-NoDerivatives 4.0 International License (CC BYNC-ND) (http://www.karger.com/Services/OpenAccessLicense). Usage and distribution for commercial purposes as well as any distribution of modified material requires written permission.
Dayse Távora-Vieira

Fiona Stanley Hospital, Outpatient Clinic 5

102-118 Murdoch Drive

Murdoch, WA 6150 (Australia)

E-Mail dayse.tavora@gmail.com 
the reason for this shift is that the $\mathrm{Cl}$ is the only device capable of restoring bilateral input to the auditory system and hence of possibly reinstating binaural hearing. Although several studies have independently shown that the $\mathrm{Cl}$ is a safe and effective treatment for SSD and AHL, clinical outcome measures in those studies and across $\mathrm{Cl}$ centers vary greatly. Only with a consistent use of defined and agreedupon outcome measures across centers can high-level evidence be generated to assess the safety and efficacy of $\mathrm{Cls}$ and alternative treatments in recipients with SSD and AHL. Methods: This paper presents a comparative study design and minimum outcome measures for the assessment of current treatment options in patients with SSD/AHL. The protocol was developed, discussed, and eventually agreed upon by expert panels that convened at the 2015 APSCl conference in Beijing, China, and at the $\mathrm{Cl} 2016$ conference in Toronto, Canada. Results: A longitudinal study design comparing CROS$\mathrm{HA}, \mathrm{BCD}$, and $\mathrm{Cl}$ treatments is proposed. The recommended outcome measures include (1) speech in noise testing, using the same set of 3 spatial configurations to compare binaural benefits such as summation, squelch, and head shadow across devices; (2) localization testing, using stimuli that rove in both level and spectral content; (3) questionnaires to collect quality of life measures and the frequency of device use; and (4) questionnaires for assessing the impact of tinnitus before and after treatment, if applicable. Conclusion: A protocol for the assessment of treatment options and outcomes in recipients with SSD and AHL is presented. The proposed set of minimum outcome measures aims at harmonizing assessment methods across centers and thus at generating a growing body of high-level evidence for those treatment options.

(C) 2017 The Author(s)

Published by S. Karger AG, Basel

\section{Introduction}

For the clinical management of single-sided deafness (SSD) or asymmetric hearing loss (AHL), the following treatment options have already received regulatory approval or are under clinical investigation: (1) a contralateral routing of signal hearing aid (CROS-HA); (2) a bone conduction device (BCD), specifically a bone-anchored hearing aid (BAHA), or (3) a cochlear implant (CI). However, despite the broader availability of at least some of these options in most countries, SSD or AHL remain untreated in the vast majority of patients.

The efficacy of each of the treatment options, including no treatment, is generally measured in terms of

1. Speech understanding in quiet

2. Speech understanding in noise

\section{Sound localization}

4. Quality of life (QoL)

5. Tinnitus reduction (when applicable)

It is important to determine what benefits (or even potential decrements) can be expected from using each device. Further, the benefits derived from each device use should be comparable across device options, including the nontreatment option. Studies have been done to this end [Arndt et al., 2017; Jacob and Stelzig, 2011; Mertens et al., 2015, 2016a, b; Távora-Vieira et al., 2013a, 2015; Van de Heyning et al., 2008; Vermeire and Van de Heyning, 2009], and systematic reviews of the available literature have found that a CI can provide adults with SSD or AHL with better speech perception in spatially separated noise, better sound localization, better QoL, and a decreased severity and incidence of tinnitus [Arndt et al., 2011; Tokita et al., 2014; van Zon et al., 2015; Vlastarakos et al., 2014] than a CROS-HA, BAHA, or nontreatment. This may not be surprising if one considers that the $\mathrm{CI}$ is the only device that reinstates the auditory input from the deaf side, hence providing the possibility to re-engage the binaural system. CROS-HAs and BCDs route the signal to the good-ear side and therefore cannot reinstate binaural hearing, nor can they suppress or mask ipsilateral tinnitus. In other words, there are a number of arguments for adults with SSD or AHL, who are willing and able to go through with the necessary postimplantation rehabilitation, to consider receiving a CI.

Although studies consistently find convincing evidence for the outcomes of CI in cases of SSD and AHL, the level of evidence remains low, primarily because different studies have used different test methodologies, thus making meaningful data comparison difficult if not impossible [Cabral Junior et al., 2016; van Zon et al., 2015]. Van Zon et al. discussed this problem in their systemic review: they were able to extract data from only 9/781 of retrieved articles and concluded that "there is a large degree of clinical heterogeneity among the studies that were included, which made it impossible to pool data" and that "larger and high-quality studies are certainly warranted before cochlear implantation can be considered as standard care."

This "clinical heterogeneity" is particularly evident in studies' variance in (1) follow-up times, (2) the tests used to assess outcomes, notably speech perception and localization outcomes, (3) whether or not a study also assessed subjects' results with a comparator device or nonintervention, and (4) the use of tests that were too easy for the subjects, resulting in ceiling effects that obscured potential device benefits. 
Previous studies were often of high quality; however, if the field of assistive hearing devices wants to clearly and robustly demonstrate the advantages (and perhaps potential disadvantages) of CI provision in adults with SSD or AHL, then future studies must be planned in such a way that will allow their data to be meaningfully compared. The purpose of this paper is to propose a simple and effective core study framework for use in SSD and AHL studies.

The need for reaching a consensus on indication criteria, assessment methods, and outcome measures for generating high-level evidence for implantable and conventional treatment options of SSD and AHL is substantiated in a number of recent publications that have proposed (1) quality standards and minimum outcome measures for every medical discipline [Koroshetz, 2015] and, in particular, for CI in adults and children [Bruce et al., 2015; Kleine Punte and Van de Heyning, 2013], (2) study protocols for the evaluation of the benefits of $\mathrm{CI}, \mathrm{BCD}$, and CROS/BiCROS-HAs in treating SSD [Kitterick et al., 2014; Peters et al., 2015a], and (3) recommendations for the identification and evaluation of CI candidates with AHL [Vincent et al., 2015]. The aim of the present paper is to propose a set of guidelines and minimum outcome measures for the evaluation of benefits (or decrements) of CI use - or the use of other conventional treatments in subjects with SSD or AHL. The proposed guidelines and outcomes measures are based, in part, on those commonly used in previous studies.

\section{Methods}

The protocol presented here is the outcome of 2 consensus meetings among clinical professionals in the fields of otorhinolaryngology and audiology from centers in Australia, Japan, India, Israel, Argentina, Canada, Belgium, the Netherlands, France, Germany, Austria, and Spain, who are experienced in the clinical management of SSD and AHL in adults and children. The first consensus meeting took place in 2015 at the 10th Asia Pacific Symposium on Cochlear Implants and Related Sciences (APSCI) in Beijing, China. The follow-up meeting took place in 2016 at the 14th International Conference on Cochlear Implants and Other Implantable Technologies (CI 2016) in Toronto, Canada.

\section{Population}

The target population is adults (18 years or older) with AHL or SSD. The criteria for the classification of SSD and AHL are defined in Table 1 and are in line with previous definitions of SSD and AHL [Vincent et al., 2015] and with the CE-approved indications for CI. Pure-tone average (PTA) is defined as the mean thresholds at pure-tone frequencies of $0.5,1,2$, and $4 \mathrm{kHz}$.

All subjects should meet all of the following inclusion criteria:

- Be 18 years of age or older at the time of enrollment

- Have SSD or AHL, as defined in Table 1
Table 1. Audiological classification criteria for AHL and SSD candidate groups

\begin{tabular}{lll}
\hline SSD & Poorer ear & PTA $\geq 70 \mathrm{~dB} \mathrm{HL}$ \\
& Better ear & PTA $\leq 30 \mathrm{~dB} \mathrm{HL}$ \\
& Interaural threshold gap & $\geq 40 \mathrm{~dB} \mathrm{HL}$ \\
\hline AHL & Poorer ear & PTA $\geq 70 \mathrm{~dB} \mathrm{HL}$ \\
& Better ear & PTA $>30$ and $\leq 55 \mathrm{~dB} \mathrm{HL}$ \\
& Interaural threshold gap & $\geq 15 \mathrm{~dB} \mathrm{HL}$ \\
\hline
\end{tabular}

SSD, single-sided deafness; AHL, asymmetric hearing loss; PTA, pure-tone average.

- Have had postlingual severe-to-profound sensorineural hearing loss in their poorer hearing ear for $<15$ years

- Do not have a deficiency of the auditory nerve, as e.g. assessed by the promontory stimulation test or magnetic resonance imaging

- Be willing and able to undertake all assessments required in the study and to go through rehabilitation training

Concerning postlingual hearing loss, most studies on SSD or AHL to date have presented evidence from cohorts with a duration of unilateral deafness of less than 10 years. However, a study in a group of CI users with long-term unilateral deafness who underwent extensive rehabilitation concludes that patients with postlingual unilateral deafness should not be excluded as CI candidates on the basis of a long duration of deafness [Távora-Vieira et al., $2013 b]$. In all subjects of this group, the duration of unilateral deafness was even greater than 15 years, which raises some doubts that there is a hard cutoff in the duration of deafness.

Potential subjects should be excluded if

- Their etiology of hearing loss contraindicates cochlear implantation

- They have tinnitus with a cause other than cochlear deafferentation, e.g. pulsatile tinnitus

- They have unrealistic expectations about possible benefits

\section{Study Design}

We propose a design which is a within-subject prospective longitudinal trial with 2 randomization groups differing in the order in which 2 initial 3-week (Bi)CROS-HA and BCD headband trial phases are conducted. At the end of the (Bi)CROS-HA and BCD trial phases, the subject should have the choice of keeping the (Bi)CROS-HA device, proceeding with BCD surgery, moving to a $\mathrm{CI}$, or refraining from any treatment altogether (Fig. 1).

We note that a cluster study design, where groups of subjects are randomly assigned to treatment options would be clearly superior. Nevertheless, some candidates who seek intervention would not consider surgery after consultation and a successful (Bi)CROS-HA trial. Other candidates might quickly identify the lack of subjective benefit of a (Bi)CROS-HA or BAHA headband and therefore opt for a CI.

In contrast to a (Bi)CROS-HA and BAHA headband trial, a CI trial is not available. Therefore, the postoperative performance of a CI and the resulting benefits are not directly accessible for the candidate. However, findings from previous studies on CI in patients with bilateral and unilateral sensorineural hearing loss allow 


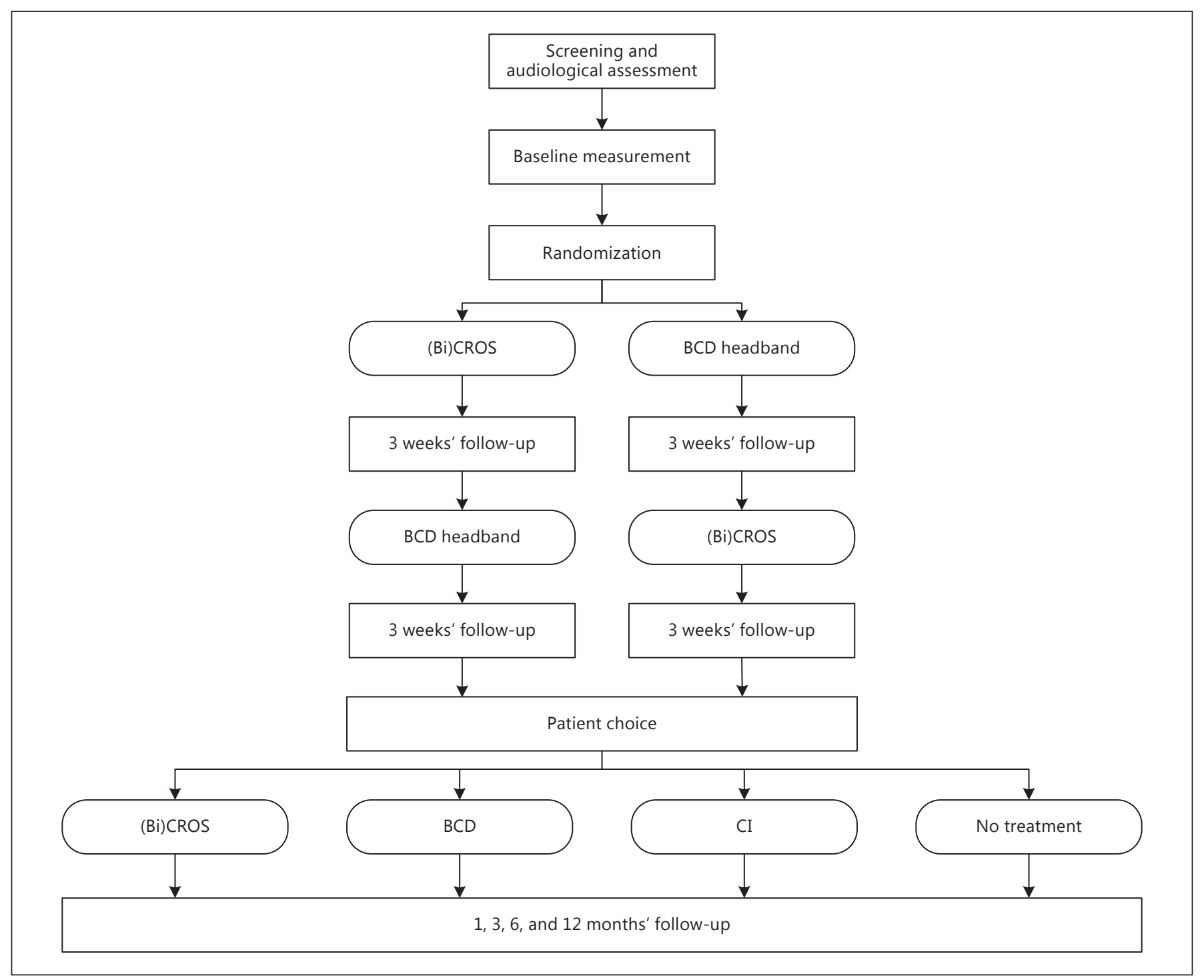

Fig. 1. Flowchart of the proposed study design.

for realistic patient counseling by the clinician - preventing unrealistic expectations and explaining the need for rehabilitation.

\section{Nomenclature}

For both SSD and AHL candidate groups, the following definitions and acronyms are used:

SSD Single-sided deaf side, i.e., poorer ear (PTA $\geq 70 \mathrm{~dB} H \mathrm{HL})$

$\mathrm{AH} \quad$ Acoustic-hearing side, i.e., better ear (PTA $\leq 30 \mathrm{~dB} H \mathrm{HL}$ in the SSD group or $>30 \mathrm{~dB} H L$ and $\leq 55 \mathrm{~dB}$ HL in the AHL group)

Unaided Listening condition with no (Bi)CROS-HA, BCD, or CI device worn on the SSD side; participants (in the AHL group) who are fitted with an HA on the contralateral ear should wear the HA; the poorer ear shall not be plugged or masked, as this does not simulate a realistic listening scenario
Aided Listening condition with the (Bi)CROS-HA, BCD, or CI device worn and activated on the SSD side; participants (in the AHL group) who are fitted with an HA on the contralateral ear should be provided with a BiCROSHA device if possible

\section{Rehabilitation}

After CI provision in cases of SSD or AHL, a systematic rehabilitation training of the implanted ear is recommended for at least 6 months after activation. The aim of this training is to facilitate the perceptual integration of the electrically stimulated ear with the dominant acoustic ear and hence the reinstatement of binaural hearing. The CI ear can be trained alone by using the CI sound processor's direct input connection or accessories for streaming audio from external devices. 
Table 2. Spatial configurations and listening conditions for speech-in-noise testing

\begin{tabular}{|c|c|c|}
\hline $\begin{array}{l}\text { Spatial } \\
\text { configuration }\end{array}$ & Listening conditions & Binaural effect measure \\
\hline $\mathrm{S}_{\mathrm{SSD}} \mathrm{N}_{\mathrm{AH}}$ & Aided, unaided & Head shadow $(\mathrm{dB})=\mathrm{SRT} \mathrm{S}_{\mathrm{SSD}} \mathrm{N}_{\mathrm{AH}}$ unaided $-\mathrm{SRT} \mathrm{S}_{\mathrm{SSD}} \mathrm{N}_{\mathrm{AH}}$ aided \\
\hline $\mathrm{S}_{0} \mathrm{~N}_{\mathrm{SSD}}$ & Aided, unaided & Squelch $(\mathrm{dB})=\mathrm{SRT} \mathrm{S} \mathrm{N}_{\mathrm{SSD}}$ unaided $-\mathrm{SRT} \mathrm{S}_{0} \mathrm{~N}_{\mathrm{SSD}}$ aided \\
\hline $\mathrm{S}_{0} \mathrm{~N}_{0}$ & Aided, unaided & Summation $(\mathrm{dB})=\mathrm{SRT} \mathrm{S}_{0} \mathrm{~N}_{0}$ unaided $-\mathrm{SRT} \mathrm{S}_{0} \mathrm{~N}_{0}$ aided \\
\hline & & $\mathrm{SRM}(\mathrm{dB})=\mathrm{SRT} \mathrm{S}_{0} \mathrm{~N}_{0}$ aided $-\mathrm{SRT} \mathrm{S}_{0} \mathrm{~N}_{\mathrm{SSD}}$ aided \\
\hline
\end{tabular}

The measures commonly used to quantify binaural effects and the way those measures are derived from the proposed testing configurations are shown in the column on the right. For all measures, a positive effect size in decibels indicates a binaural benefit. SSD, single-sided deafness; AH, acoustic hearing; SRT, speech reception threshold; SRM, spatial release from masking.

\section{Minimum Outcome Measures}

Outcome measures should be collected at each of the following study intervals: at baseline, after each of the initial 3-week (Bi) CROS-HA and BCD headband trials, and at 1, 3, 6, and 12 months after device activation or nontreatment.

The following outcome measures should be collected: audiological assessment via PTA; speech perception in noise; sound localization; QoL; if applicable, tinnitus, and amount of daily device use.

\section{Pure-Tone Audiogram}

At the initial screening, the audiological assessment should include the measurement of air conduction hearing thresholds (PTA averaged over frequencies of $0.5,1,2$, and $4 \mathrm{kHz}$ ) in both ears. The assessments should be conducted using a standard audiological setup in a sound-treated room. Stimuli should be presented via headphones with the better ear masked using insert ear phones. Participants can then be stratified into the SSD or AHL group according to the criteria defined in Table 1.

On all subsequent follow-up visits, PTA thresholds should be measured in the better ear. Monitoring hearing thresholds is important for the subjects in the AHL group, to ensure they still match the criteria for AHL, and for subjects in the SSD group, to ensure that their hearing levels remain within the normal range throughout the study. Since progressive hearing loss might occur in either group, this aspect has to be dealt with separately during data analysis. Over the duration of the study, speech perception scores as well as other outcome measures might decrease due to a progressive hearing loss in the better ear. Therefore, in order to demonstrate the added value of either intervention, we consider it essential to track the aided and unaided (CROS, BCD, or CI deactivated) performance measures at all study intervals.

Masked speech perception

Masked speech perception should be assessed with a standard audiometric and validated sentence test, using a free-field setup in a sound-treated room. The masker should be presented at a fixed level of $65 \mathrm{dBA}$, following common practice in audiometric testing. The level of the target signal should be adapted to measure the speech reception threshold at which $50 \%$ of the sentences are understood correctly. If possible, same-gender 2-talker babble should be used as the masking signal, because it has been shown to be (1) a more effective speech masker than other noise signals and (2) more sensitive in demonstrating benefits of a CI or a second good ear over a first good ear [Bernstein et al., 2016].

The recommended spatial configurations and listening conditions are outlined in Table 2. Head shadow, summation, binaural squelch, and spatial release from masking are measures of binaural benefit that can be derived from the proposed testing configurations [Gartrell et al., 2014]. Test time adds up to 30-45 min for all spatial configurations and listening conditions, assuming 5-7 min per test list.

The spatial configuration $\mathrm{S}_{\mathrm{SSD}} \mathrm{N}_{\mathrm{AH}}$ assesses the effect of the head shadow on speech recognition by (1) presenting the speech signal to the side of the poorer ear and the masker to the side of the better ear and (2) by comparing speech reception thresholds for the aided condition with those of the unaided listening condition. The detrimental head shadow effect is overcome with the addition of a CROS-HA, BCD headband, or CI device on the poor-ear side (i.e., the side with the better signal-to-noise ratio).

In the spatial configuration $\mathrm{S}_{0} \mathrm{~N}_{\mathrm{SSD}}$, the signal is presented from the front and the masker from the side of the poorer ear. In the aided condition, the CROS-HA, BCD, or CI device receives the more adverse target-to-masker ratio (TMR). While the binaural squelch still produces a benefit of approximately $3 \mathrm{~dB}$ in normalhearing listeners [Bronkhorst and Plomp, 1988], CROS-HA and BCD devices consistently show a decrement in speech recognition in this configuration [Peters et al., 2015b] because they essentially route the masker to the better ear and therefore reduce the effective TMR ratio on that side. CI users with SSD or AHL, however, have consistently been found to perform equally or better with the CI activated in this configuration, suggesting that a CI is able to reinstate binaural hearing [van Zon et al., 2015].

With the spatial configuration $\mathrm{S}_{0} \mathrm{~N}_{0}$, the effect of binaural summation on speech recognition is assessed by presenting both the signal and the masker from the front and by comparing speech reception thresholds for the aided condition with those of the unaided listening condition.

\section{Sound Localization}

Sound localization ability should be assessed using the setup shown in Figure 2. Localization testing should be conducted in a sound-treated room with at least 7 loudspeakers equally distributed along a semicircle between $-90^{\circ}$ (left) and $90^{\circ}$ (right) azimuth. 


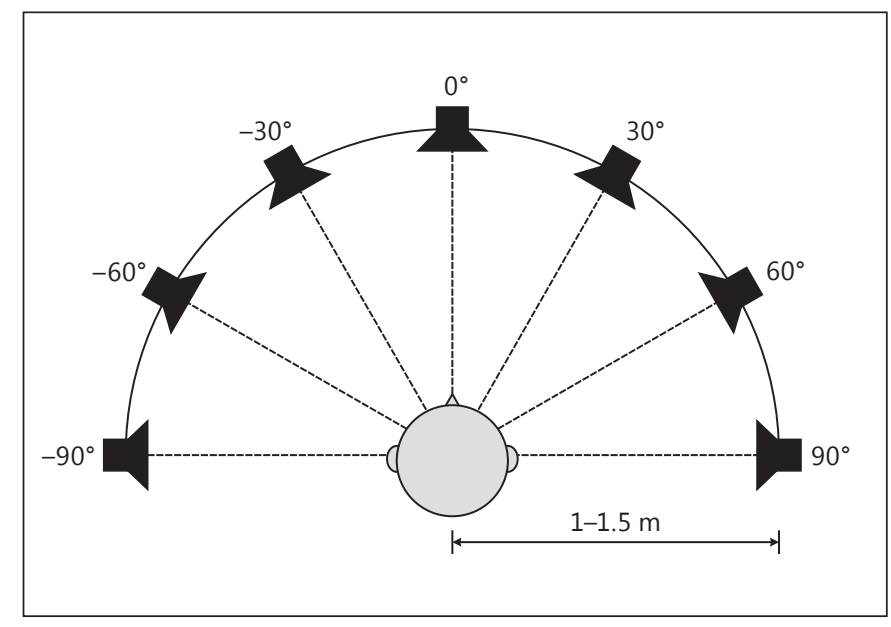

Fig. 2. Test setup for sound localization measurement. The radius of the loudspeaker ring should be $1.5 \mathrm{~m}$, if possible, but at least $1 \mathrm{~m}$.

Thus, the angle between 2 adjacent loudspeakers would be $30^{\circ}$. Seven loudspeakers can be controlled by 1 commercially available 8 -channel sound card and are sufficient to resolve localization performance down to $9^{\circ}$ over a loudspeaker span of $180^{\circ}$ [Hartmann et al., 1998]. The recommended radius of the semicircle is $1.5 \mathrm{~m}$ and should be at least $1 \mathrm{~m}$, with the loudspeakers at the level of a hypothetical plane going through the subject's ear canals.

The recommended localization stimuli are single noise bursts with a duration of $1 \mathrm{~s}$, including rise and fall times of $20 \mathrm{~ms}$ each. Two noise stimuli of different spectral shapes [Schoen et al., 2005] should be presented randomly in order to confound monaural spectral cues. The head acts as an acoustic barrier that more effectively attenuates high frequencies than low frequencies. Therefore, in a monaural listening condition, the perceived "brightness" of the same signal will be distinctly different due to differences in coloration when presented from opposite directions, i.e., a signal that sounds bright when presented from the ipsilateral side sounds muffled when presented from the contralateral side. If the spectrum of the localization stimulus is not varied, these monaural spectral cues can be assigned to direction and result in an overestimated monaural localization performance. The 2 spectrally shaped noise stimuli are generated by filtering a CCITT noise stimulus from a virtual sound source located at $90^{\circ}$ azimuth (right-ear side) using the ipsilateral and contralateral head-related transfer functions from the MIT diffuse-field-equalized Kemar head-related transfer function data set [Gardner and Martin, 1994]. The magnitude spectra of the resulting noise stimuli are shown in Figure 3.

Similarly to spectral cues, loudness cues will also systematically vary as a function of source direction if the stimulus level is kept constant. In a monaural listening condition, sounds presented ipsilaterally are perceived as louder than the same sounds presented centrally. Likewise, the loudness of a signal can be assigned to directions and overestimate monaural localization performance. Therefore, localization stimuli should be presented randomly at 1 of 3 levels $(65,70$, or $75 \mathrm{dBA})$. The used levels span a range which is also produced by moving a sound source with a fixed level from

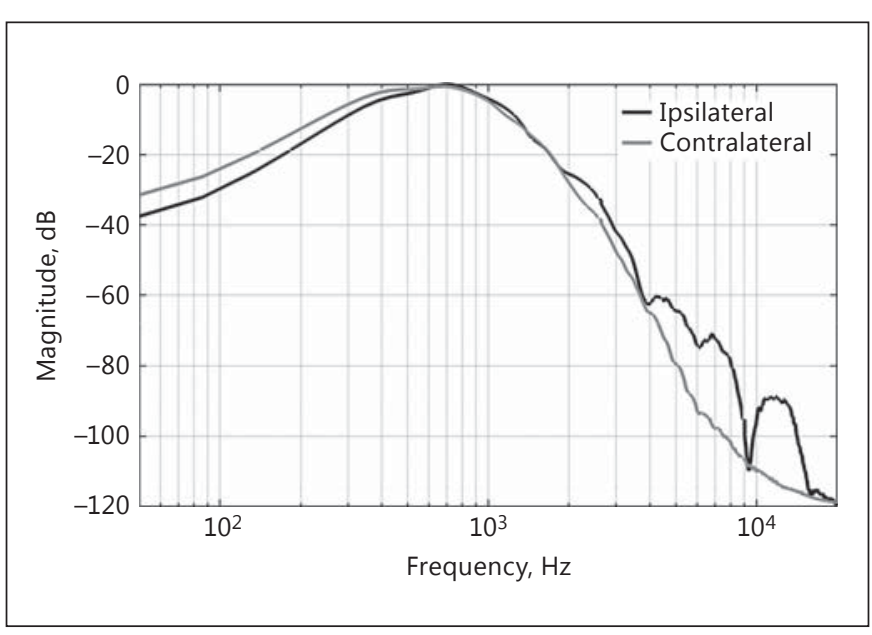

Fig. 3. Magnitude responses of spectrally shaped CCITT noise stimuli used for localization.

left to right and resolve the covariance of direction and unilaterally perceived sound level.

Each stimulus is presented once, resulting in a total of 42 stimulus presentations (7 loudspeakers $\times 3$ levels $\times 2$ signals) and an estimated testing time of about 2-3 min per listening condition. The presentation level, the signal type, and the loudspeaker a stimulus is presented from are randomized. Subjects are instructed not to turn their head during stimulus presentation. No feedback regarding correct or incorrect responses is given, to prevent learning of remaining direction-covariant acoustic features (e.g. room acoustics, reflections, and loudspeaker frequency responses). Localization performance should be quantified as the total root mean square error of the speaker angles indicated by the subject and the speaker angles the stimuli were presented from. A lower root mean square error indicates better localization skills. Furthermore, the localization bias should be evaluated by averaging all response azimuths. A small bias indicates a centered auditory image.

\section{QoL and Tinnitus}

Impact of Hearing Loss

The speech, spatial, and qualities of hearing (SSQ) questionnaire should be used in its shorter 12-question version [Noble et al., 2013]. The short version of the SSQ questionnaire provides similar results to the full version comprising 49 questions. A conversion formula between the abbreviated and full versions has been provided by Noble et al. [2013]. The SSQ questionnaire is a sensitive and specific measure to assess the impact of hearing loss on speech perception, sound localization, and QoL. Most studies investigating the benefits of the various treatment options for SSD or AHL have utilized the SSQ as the primary instrument for assessing subjective outcomes in the subdomains addressed by the questionnaire.

Generic QoL Questionnaire

As a generic measure of general health status, the Health Utilities Index Mark 3 [Horsman et al., 2003] should be used to 
Table 3. Summary of outcome measures collected over time

\begin{tabular}{lllllll}
\hline Outcome measure & Baseline & After each & \multicolumn{4}{l}{ Months after device provision or nontreatment } \\
\cline { 5 - 7 } & & 3-week trial & 1 & 3 & 6 & 12 \\
\hline PTA & both ears & better ear & better ear & better ear & better ear & better ear \\
Speech perception in noise & yes & yes & yes & yes & yes & yes \\
Sound localization & yes & yes & yes & yes & yes & yes \\
QoL & yes & yes & yes & yes & yes & yes \\
Tinnitus, if applicable & yes & yes & yes & yes & yes & yes \\
Daily device use & - & yes & yes & yes & yes & yes \\
\hline
\end{tabular}

evaluate a single index value of the health status. The Health Utilities Index Mark 3 is the standard instrument for health technology assessments evaluating the cost-effectiveness of a treatment.

\section{Tinnitus Questionnaire}

In subjects with tinnitus of the types falling into the $\mathrm{CI}$ indication criteria as put forward by Van de Heyning et al. [2008], the tinnitus functional index questionnaire [Meikle et al., 2012] should be used to assess the handicap from tinnitus at the preoperative interval and at all follow-up intervals. In addition to the tinnitus functional index, a visual-analog scaling of tinnitus loudness should also be completed.

\section{Device Use Time}

A "daily use criterion" that reflects the hours of consistent device use should be included in the longitudinal data collection. This should be done either via a simple questionnaire of hours of use per day or technical options such as data logging functions in the worn device. Table 3 summarizes all outcome measures collected over the course of the proposed study design.

\section{Conclusion}

Hearing professionals and their patients would benefit from the broad adoption of a standard protocol for testing adults with SSD and AHL. Such a protocol would allow results to be compared and the advantages and disadvantages of various treatments to be more clearly seen. The protocol proposed herein is consistent, thorough, and may be completed by clinics within the range of their normally available resources.

\section{References}

Arndt S, Aschendorff A, Laszig R, Beck R, Schild C, Kroeger S, Ihorst G, Wesarg T: Comparison of pseudobinaural hearing to real binaural hearing rehabilitation after cochlear implantation in patients with unilateral deafness and tinnitus. Otol Neurotol 2011;32:39-47.

Arndt S, Laszig R, Aschendorff A, Hassepass F, Beck R, Wesarg T: Cochlear implant treatment of patients with single-sided deafness or asymmetric hearing loss. HNO 2017, Epub ahead of print.

Bernstein JG, Goupell MJ, Schuchman GI, Rivera AL, Brungart DS: Having two ears facilitates the perceptual separation of concurrent talkers for bilateral and single-sided deaf cochlear implantees. Ear Hear 2016;37:289-302.

Bronkhorst AW, Plomp R: The effect of head-induced interaural time and level differences on speech intelligibility in noise. J Acoust Soc Am 1988;83:1508-1516.
Bruce I, Cooper H, Waltzman S, Schramm D, Graham J: Editorial maximising research value in the field of hearing implantation: a call for "big data". Cochlear Implants Int 2015;16: 301-302.

Cabral Junior F, Pinna MH, Alves RD, Malerbi AF, Bento RF: Cochlear implantation and single-sided deafness: a systematic review of the literature. Int Arch Otorhinolaryngol 2016;20:69-75.

Gardner WG, Martin KD: HRTF measurements of a Kemar dummy-head microphone. 1994. http://sound.media.mit.edu/resources/ KEMAR.html.

Gartrell BC, Jones HG, Kan A, Buhr-Lawler M, Gubbels SP, Litovsky RY: Investigating longterm effects of cochlear implantation in single-sided deafness: a best practice model for longitudinal assessment of spatial hearing abilities and tinnitus handicap. Otol Neurotol 2014;35:1525-1532.
Hartmann WM, Rakerd B, Gaalaas JB: On the source-identification method. J Acoust Soc Am 1998;104:3546-3557.

Horsman J, Furlong W, Feeny D, Torrance G: The health utilities index (HUI): concepts, measurement properties and applications. Health Qual Life Outcomes 2003;1:54.

Jacob R, Stelzig Y: The Koblenz experience in treating single-sided deafness with cochlear implants. Audiol Neurootol 2011;16(suppl 1): 6-8.

Kitterick PT, O'Donoghue GM, EdmondsonJones M, Marshall A, Jeffs E, Craddock L, Riley A, Green K, O'Driscoll M, Jiang D, Nunn T, Saeed S, Aleksy W, Seeber BU: Comparison of the benefits of cochlear implantation versus contra-lateral routing of signal hearing aids in adult patients with single-sided deafness: study protocol for a prospective within-subject longitudinal trial. BMC Ear Nose Throat Disord 2014;14:7. 
Kleine Punte A, Van de Heyning P: Quality standards for minimal outcome measurements in adults and children. Cochlear Implants Int 2013;14(suppl 2):S39-S42.

Koroshetz W: A core set of trial outcomes for every medical discipline? BMJ 2015;350:h85.

Meikle MB, Henry JA, Griest SE, Stewart BJ, Abrams HB, McArdle R, Myers PJ, Newman CW, Sandridge S, Turk DC, Folmer RL, Frederick EJ, House JW, Jacobson GP, Kinney SE, Martin WH, Nagler SM, Reich GE, Searchfield G, Sweetow R, Vernon JA: The tinnitus functional index: development of a new clinical measure for chronic, intrusive tinnitus. Ear Hear 2012;33:153-176.

Mertens G, Kleine Punte A, De Bodt M, Van de Heyning P: Binaural auditory outcomes in patients with postlingual profound unilateral hearing loss: 3 years after cochlear implantation. Audiol Neurootol 2015;20(suppl 1):6772.

Mertens G, De Bodt M, Van de Heyning P: Cochlear implantation as a long-term treatment for ipsilateral incapacitating tinnitus in subjects with unilateral hearing loss up to 10 years. Hear Res 2016a;331:1-6.

Mertens G, Desmet J, De Bodt M, Van de Heyning P: Prospective case-controlled sound localization study after cochlear implantation in adults with single-sided deafness and ipsilateral tinnitus. Clin Otolaryngol 2016b;41:511518.
Noble W, Jensen NS, Naylor G, Bhullar N, Akeroyd MA: A short form of the speech, spatial and qualities of hearing scale suitable for clinical use: the SSQ12. Int J Audiol 2013;52:409412.

Peters JPM, van Zon A, Smit AL, van Zanten GA, de Wit AG, Stegeman I, Grolman W: CINGLE-trial: cochlear implantation for singlesided deafness, a randomised controlled trial and economic evaluation. BMC Ear Nose Throat Disord 2015a;15:1-8.

Peters JP, Smit AL, Stegeman I, Grolman W: Review: bone conduction devices and contralateral routing of sound systems in single-sided deafness. Laryngoscope 2015b;125:218-226.

Schoen F, Mueller J, Helms J, Nopp P: Sound localization and sensitivity to interaural cues in bilateral users of the Med-El Combi 40/40+ cochlear implant system. Otol Neurotol 2005; 26:429-437.

Távora-Vieira D, Marino R, Krishnaswamy J, Kuthbutheen J, Rajan GP: Cochlear implantation for unilateral deafness with and without tinnitus: a case series. Laryngoscope 2013a;123:1251-1255.

Távora-Vieira D, Boisvert I, McMahon CM, Maric V, Rajan GP: Successful outcomes of cochlear implantation in long-term unilateral deafness: brain plasticity? Neuroreport 2013b;24:724-729.

Távora-Vieira D, Marino R, Acharya A, Rajan GP: The impact of cochlear implantation on speech understanding, subjective hearing performance, and tinnitus perception in patients with unilateral severe to profound hearing loss. Otol Neurotol 2015;36:430-436.
Tokita J, Dunn C, Hansen MR: Cochlear implantation and single-sided deafness. Curr Opin Otolaryngol Head Neck Surg 2014;22:353358.

Van de Heyning P, Vermeire K, Diebl M, Nopp P, Anderson I, De Ridder D: Incapacitating unilateral tinnitus in single-sided deafness treated by cochlear implantation. Ann Otol Rhinol Laryngol 2008;117:645-652.

Van Zon A, Peters JP, Stegeman I, Smit AL, Grolman W: Cochlear implantation for patients with single-sided deafness or asymmetrical hearing loss: a systematic review of the evidence. Otol Neurotol 2015;36:209-219.

Vermeire K, Van de Heyning P: Binaural hearing after cochlear implantation in subjects with unilateral sensorineural deafness and tinnitus. Audiol Neurootol 2009;14:163-171.

Vincent C, Arndt S, Firszt JB, Fraysse B, Kitterick PT, Papsin BC, Snik A, Van de Heyning P, Deguine O, Marx M: Identification and evaluation of cochlear implant candidates with asymmetrical hearing loss. Audiol Neurootol 2015;20(suppl 1):87-89.

Vlastarakos PV, Nazos K, Tavoulari EF, Nikolopoulos TP: Cochlear implantation for singlesided deafness: the outcomes. An evidencebased approach. Eur Arch Otorhinolaryngol 2014;271:2119-2126. 